\title{
Spotlight on bevacizumab in metastatic colorectal cancer: patient selection and perspectives
}

This article was published in the following Dove Press journal:

Gastrointestinal Cancer:Targets and Therapy

30 June 2016

Number of times this article has been viewed

\section{Manojkumar Bupathi \\ Daniel H Ahn \\ Tanios Bekaii-Saab}

Department of Medical Oncology, Richard Solove Research Institute and James Cancer Hospital, The Ohio State University Wexner Medical Center, Columbus, $\mathrm{OH}$, USA
Correspondence: Tanios Bekaii-Saab Department of Medical Oncology, Richard Solove Research Institute and James Cancer Hospital, The Ohio State University - James Cancer Hospital, A454 Starling Loving Hall, 320 West 10th Avenue, Columbus, OH 43210, USA $\mathrm{Tel}+\mathrm{I} 6142939863$

Email Tanios.Saab@osumc.edu

\begin{abstract}
Metastatic colorectal cancer $(\mathrm{mCRC})$ is a prevalent disease for which combination cytotoxic chemotherapy is the mainstay of treatment. With the use of targeted therapy, including anti-angiogenic agents, there have been significant improvements in overall outcome of patients with mCRC. Bevacizumab, a monoclonal antibody targeting the vascular endothelial growth factor ligand A, is approved for use in $\mathrm{mCRC}$ patients in both the first and second lines of therapy. With a better understanding of the disease through molecular profiling, identification of prognostic biomarkers may lead to better patient selection with improved outcomes for those affected by this disease.
\end{abstract}

Keywords: VEGF, colon, rectum, cancer

\section{Introduction}

Colorectal cancer (CRC) is the third most common cancer and the third leading cause of cancer-related deaths in the US. ${ }^{1}$ In 2014, there were 136,830 new cases of CRC and 50,310 CRC-related deaths in the US. ${ }^{1}$ While patients diagnosed with early-stage CRC (stage I-III) have good long-term outcomes with 5-year survival exceeding $50 \%,{ }^{1}$ those diagnosed with metastatic disease have poor outcomes with a 5-year survival rate that remains under $12 \%{ }^{2}$ However, over the past several years, advancements in therapeutic approaches have led to an improvement in patient outcomes with a median overall survival (OS) approaching 3 years. This is largely due to the increase in availability of active regimens with the incorporation of targeted therapy, including anti-angiogenic agents.

\section{Background on VEGF inhibition}

Angiogenesis is a mechanism by which tumor cells can proliferate and is controlled by vascular endothelial growth factor (VEGF), primarily VEGF-A (Figure 1). Elevated levels of VEGF-A are associated with metastatic disease of the liver and lymph nodes., Furthermore, in addition to its association with an overall poor prognosis, findings suggest that expression levels may be predictive of more aggressive distant metastatic disease. $^{5-7}$ Takahashi et $\mathrm{al}^{7}$ showed that the expression of VEGF and its receptor was higher in metastatic disease, correlated with time to progression. ${ }^{8}$ VEGF targeting agents have been combined with other chemotherapy agents, leading to improved outcomes in several solid tumor malignancies, including gastric, lung, colon, rectum, and ovary. ${ }^{9}$ Based on multiple large, randomized Phase III studies, there are three 


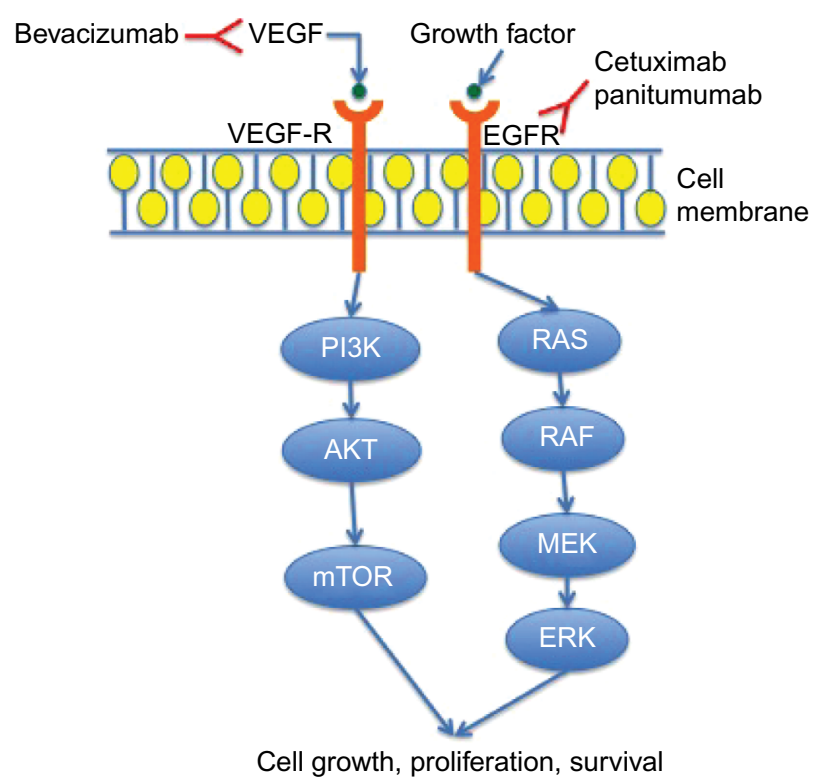

Figure I The signaling cascade of VEGF and EGFR.

Note: The red antibodies indicate the point of action of anti-VEGF and anti-EGFR therapy.

Abbreviations: VEGF, vascular endothelial growth factor; VEGF-R, vascular endothelial growth factor receptor; EGFR, epidermal growth factor receptor; PI3K, phosphoinositide 3-kinase.

approved anti-VEGF therapies in metastatic colorectal cancer (mCRC): bevacizumab, ziv-aflibercept, and ramucirumab. ${ }^{10-12}$

\section{Role of RAS in choice of therapy}

Epidermal growth factor receptor (EGFR) is a transmembrane tyrosine kinase, which binds to different ligands (Figure 1). ${ }^{13}$ Upon binding to its receptor, the structure is changed leading to downstream autophosphorylation and activation of intracellular signaling cascade, specifically the mitogen-activated protein kinase and phosphoinositide 3-kinase pathways. Activation and downstream phosphorylation of the mitogenactivated protein kinase and phosphoinositide 3-kinase pathways lead to tumor cell proliferation, anti-apoptosis, activation of invasion and metastasis, and tumor-induced neovascularization. ${ }^{14}$

EGFR is an important therapeutic target in patients with mCRC but is only active in a specified subset of patients. $K R A S$, a proto-oncogene, is a downstream signaling component of EGFR that is involved in cell proliferation, apoptosis, and tumor-induced neoangiogenesis (Figure 1). One-third of patients with CRC have a mutation in exon 2 of $K R A S$, and $15 \%$ were found to have an additional mutation at exons 3 and 4 of KRAS and exons 2, 3, and 4 of NRAS. ${ }^{15-17}$ Given these findings, it is now recommended that extended $R A S$ testing be done for all patients prior to initiation of first-line therapy or at least prior to initiation of anti-EGFR therapy. Further, $K R A S$ and NRAS mutational status has been shown to predict the outcome of patients treated with anti-EGFR agents. ${ }^{18} \mathrm{mCRC}$ patients who are wild-type (WT) for $R A S$ have had improvement in OS, progression-free survival (PFS), and response rate (RR) when treated with cetuximab or panitumumab as single agent or in combination with chemotherapy. ${ }^{19,20} \mathrm{By}$ contrast, patients who have $R A S$ mutations and were treated with anti-EGFR therapy tend to have worse outcomes. WT $R A S$ is predictive of response to anti-EGFR therapy compared with $R A S$-mutated patients who do not respond. Furthermore, it is clear that patients with a $B R A F \mathrm{~V} 600 \mathrm{E}$ mutation $(\sim 5 \%$ of all patients) have a poor prognosis regardless of the type of treatment they receive, except for those who have microsatellite high tumors. The predictive value of BRAF mutations and response to anti-EGFR therapy have been controversial. ${ }^{21,22}$ Neither $R A S$ nor $B R A F$ mutations have any predictive role for bevacizumab or other VEGF inhibitors.

\section{Treatment options of metastatic disease}

\section{The role of chemotherapy}

The OS for patients with untreated mCRC is $\sim 6$ months; however, with the use of combinations of cytotoxic chemotherapy and targeted agents, this has been improved to close to 3 years from a historical 12-month period with 5-fluorouracil (5-FU) alone. ${ }^{23}$ The primary backbone of therapy for mCRC patients is 5-FU, which is a pyrimidine analog that interrupts DNA and RNA synthesis. 5-FU is normally administered as a bolus with leucovorin (LV), which is a folate analog that stabilizes thymidylate synthase and enhances the activity of 5-FU. 5-FU/LV has efficacy for patients with $\mathrm{mCRC}$; however, in the metastatic setting when it is combined with a platinum derivative, oxaliplatin, or a topoisomerase inhibitor, irinotecan (IFL), there are improvements in patients' PFS and OS. One of the first studies to demonstrate an advantage for combination chemotherapy is that of bolus 5-FU and IFL that was associated with an improved RR and OS compared to bolus 5-FU alone (49\% vs $31 \%[P<0.001]$ and 17.4 vs 14.1 months $[P=0.031]$, respectively) ${ }^{24}$ Another study demonstrated that the combination of infusional 5-FU with oxaliplatin (FOLFOX) led to an improvement in median PFS and RR when compared to 5-FU (9.0 vs 6.2 months $[P=0.0003]$ and $50.7 \%$ vs $22.3 \%$ $[P=0.0001]$, respectively). ${ }^{25}$ Unfortunately, this study did not demonstrate a clear survival advantage. The combination of all the three agents was explored with 5-FU, oxaliplatin, and IFL (FOLFOXIRI) demonstrated an improvement in RR, OS, and PFS when compared to infusional 5-FU and IFL ( $60 \%$ vs $34 \%, P<0.0001$; median OS 22.6 vs 16.7 months, 
hazard ratio [HR] $0.70, P=0.032$; median PFS 9.8 vs 6.9 months, HR 0.63, $P=0.0006) .{ }^{26}$ Wider utilization of this regimen has been limited by its potential for significant toxicities.

\section{The role of bevacizumab in the first-line treatment of $\mathrm{mCRC}$}

Bevacizumab is a humanized monoclonal antibody that binds to and neutralizes all human VEGF-A. This agent was first introduced in clinical trials in 1997. The first Phase I study suggested that bevacizumab as a single agent was relatively non-toxic and combining it with standard chemotherapy did not seem to exacerbate chemotherapy-related toxicities. ${ }^{27,28}$ Bevacizumab was investigated at different schedules and in combinations with a variety of chemotherapeutic regimens (5-FU, FOLFIRI, FOLFOX, or FOLFOXIRI). Bevacizumab combined with chemotherapy in the first-line setting demonstrated an improvement in the outcome of patients with mCRC. ${ }^{29}$ First, Kabbinavar et al conducted a randomized Phase II study to evaluate the efficacy and safety of bevacizumab with 5-FU/LV. ${ }^{29}$ In this three-arm study, a total of 104 enrolled patients were randomized to receive the following: 1) 5-FU/LV (control arm), 2) 5-FU/LV + bevacizumab at $5 \mathrm{mg} / \mathrm{kg}$, or 3) $5-\mathrm{FU} / \mathrm{LV}+$ bevacizumab at $10 \mathrm{mg} / \mathrm{kg}$. Compared to the control arm, patients who received bevacizumab, regardless of the dose, experienced higher RRs and longer median time to disease progression and OS (lowdose arm: 40\%, PFS 9 months, OS 21.5 months; high-dose arm: 24\%, PFS 7.2 months, OS 16.1 months). ${ }^{29}$

\section{Bevacizumab in combination with doublet chemotherapy}

Based on prior results, the combination of bevacizumab with 5-FU/LV is active in patients with $\mathrm{mCRC}$. It was also known that IFL with 5-FU/LV improves outcomes in mCRC patients. ${ }^{30}$ From this, Hurwitz et al conducted a Phase III randomized trial. ${ }^{31}$ In the study, IFL + bevacizumab (bev) was investigated in 813 patients with advanced CRC. Patients were randomized and received IFL + placebo or IFL + bev. Findings demonstrated significant improvements in OS, PFS, and RR in patients who received bev (20.3 vs 15.6 months, HR $0.66, P<0.001 ; 10.6$ vs 6.2 months, HR $0.54, P<0.001 ; 44.8 \%$ vs $34.8 \%, P=0.004$, respectively). ${ }^{31}$ Another key trial that evaluated bevacizumab with doublet chemotherapy was Eastern Cooperative Oncology Group 3200 study. ${ }^{32}$ This study enrolled patients who were previously treated with IFL and found that OS (12.9 vs 10.8 months; $P<0.0011$ ), PFS (7.3 vs 4.7 months; $P<0.0001$ ), and RR $(22.7 \%$ vs $8.6 \% ; P<0.0001)$ were significantly better with FOLFOX/bevacizumab compared to FOLFOX alone. Based on these results, bevacizumab was eventually approved for first- and second-line treatments in patients with bevacizumab-naïve mCRC.

Given the improved toxicity profile and likely improved outcomes, regimens including infusional 5-FU were preferred over bolus 5-FU to be used in combination therapy. ${ }^{33}$ Fuchs et al conducted a randomized study evaluating three different IFL-containing regimens in first-line mCRC: 1) FOLFIRI with bevacizumab, 2) IFL in combination with bolus 5-FU/LV (mIFL) with bevacizumab, and 3) IFL plus capecitabine. ${ }^{34}$ Patients who received FOLFIRI with bevacizumab had a significantly greater OS compared to those who received mIFL with bevacizumab (median OS, 19.2 months; $P=0.007 ; \mathrm{HR}$ for death $=2.34 ; 95 \%$ confidence interval $[\mathrm{CI}]$ 1.34-4.12). ${ }^{34}$ Additionally, the combination of FOLFIRI with bevacizumab had an improved toxicity profile. ${ }^{34} \mathrm{~A}$ similar design with oxaliplatin-based therapy was conducted by Hochster et al. ${ }^{35}$ The TREE-2 trial was a Phase II study that randomized treatment-naïve $\mathrm{mCRC}$ patients to bevacizumab with different fluoropyrimidines and oxaliplatin regimens. The study demonstrated a median OS of 26.1 months and RR of $52 \%$ with tolerable adverse effects. ${ }^{35}$ It is also suggested that a bolus 5-FU regimen is less desirable than an infusional regimen. A larger randomized Phase III study by Saltz et al suggested that the combination of bevacizumab with first-line chemotherapy (either capecitabine plus oxaliplatin or 5-FU/ folinic acid plus oxaliplatin), compared to placebo, improved PFS (9.4 vs 8.0 months; HR 0.83 ; $97.5 \%$ CI $0.72-0.95$; $P=0.0023)$, however, with no significant differences in OS or RR. ${ }^{36}$ The lack of OS and RR benefit was postulated to be secondary to the inability of bevacizumab to be continued at the time of progression.

Most recently, the MAVERICC trial, a large Phase II randomized study, evaluated mFOLFOX6 with bevacizumab vs FOLFIRI with bevacizumab as first-line chemotherapy in patients with treatment-naïve mCRC. Outcome results from this study suggest that FOLFIRI may have an edge over FOLFOX when combined with bevacizumab, although both the regimens remain acceptable in this setting (Table 1). ${ }^{37}$

\section{Bevacizumab with triplet chemotherapy}

A combination of 5-FU, oxaliplatin, and IFL, FOLFOXIRI, was initially evaluated with bevacizumab in a Phase 2 study and showed interesting outcomes with a RR of $77 \%$, median PFS of 13.1 months, and median OS of 30.9 months. ${ }^{38}$ Based on these results, a randomized Phase III study was conducted evaluating FOLFOXIRI with bevacizumab in comparison to 
Table I Bevacizumab as first-line therapy

\begin{tabular}{|c|c|c|c|c|c|}
\hline Study & Phase & Treatment arms & PFS (months) & OS (months) & $P$-value \\
\hline \multirow[t]{2}{*}{ Hurwitz et al $\left.\right|^{11,31}$} & III & IFL/Bevacizumab & 10.6 & 20.3 & $<0.001$ \\
\hline & & IFL/Placebo & 6.2 & 15.6 & NR \\
\hline \multirow[t]{5}{*}{ Fuchs et $\mathrm{al}^{10,34}$} & III & FOLFIRI & 7.6 & 23.1 & NR \\
\hline & & $\mathrm{mIFL}$ & 5.9 & 17.6 & NR \\
\hline & & CapelRI & 5.8 & 18.9 & NR \\
\hline & & FOLFIRI/Bevacizumab & NR & Not reached & NR \\
\hline & & mIFL/Bevacizumab & NR & 19.2 & NR \\
\hline \multirow[t]{2}{*}{ Saltz et al ${ }^{36 \#}$} & III & XELOX or FOLFOX/placebo & 8.0 & 19.9 & 0.077 \\
\hline & & XELOX or FOLFOX/bevacizumab & $9.4^{*}$ & 21.3 & NR \\
\hline \multirow[t]{3}{*}{ Hochster et a $\left.\right|^{35}$} & III & mFOLFOX6/Bevacizumab & NR & 26.1 & NR \\
\hline & & FOLFOX/Bevacizumab & NR & 20.4 & NR \\
\hline & & CapOx/Bevacizumab & NR & 24.6 & NR \\
\hline \multirow[t]{2}{*}{ Kabbinavar et a ${ }^{29}$} & II & 5-FU + LV/Bevacizumab & $9.2^{*}$ & 16.6 & 0.16 \\
\hline & & 5-FU + LV/Placebo & 5.5 & 12.9 & NR \\
\hline \multirow[t]{3}{*}{ Tebbutt et $\mathrm{al}^{43}$} & III & Capecitabine & 5.7 & 18.9 & 0.001 \\
\hline & & $\mathrm{CB}$ & 8.4 & NR & NR \\
\hline & & CBM & 8.4 & 16.4 & NR \\
\hline Masi et $\mathrm{al}^{38}$ & II & FOLFOXIRI/Bevacizumab & 13.1 & 30.9 & NR \\
\hline \multirow[t]{2}{*}{ Loupakis et a ${ }^{39}$} & II & FOLFIRI/Bevacizumab & 9.7 & 25.8 & 0.003 \\
\hline & & FOLFOXIRI/Bevacizumab & $12.1 *$ & 31.0 & NR \\
\hline \multirow[t]{2}{*}{ Lenz et $\mathrm{al}^{37}$} & II & mFOLFOX6/bev & 10.1 & 23.9 & NS \\
\hline & & FOLFIRI/bev & 12.6 & 27.5 & NR \\
\hline
\end{tabular}

Notes: *PFS was statistically significant in this study; "OS was not statistically significant. In this study, OS was reported with capecitabine, mitomycin, and bevacizumab. NS, both PFS and OS were not significant in this study.

Abbreviations: PFS, progression-free survival; OS, overall survival; IFL, irinotecan; 5-FU, 5-fluorouracil; LV, leucovorin; CB, capecitabine plus bevacizumab; CBM, capecitabine, bevacizumab, and mitomycin; bev, bevacizumab; NR, not reported; NS, not significant; CapelRI, capecitabine plus irinotecan.

FOLFIRI with bevacizumab (TRIBE). A total of 508 patients with untreated $\mathrm{mCRC}$ were enrolled in either treatment arm. The median PFS was 12.1 months in FOLFOXIRI with bevacizumab compared to 9.7 months in FOLFIRI with bevacizumab. In addition there was an improved RR in the FOLFOXIRI with bevacizumab group (65\% vs 53\%). However, this regimen had a significantly higher toxicity profile when compared to FOLFIRI with bevacizumab, and OS was not significantly different between both the groups. ${ }^{39}$ Additionally, there were no meaningful differences in the liver resection or $\mathrm{R} 0$ resection rates between the two arms.

To investigate further the potential benefits of adding bevacizumab to a triplet drug combination vs a doublet, STEAM was designed as a Phase II randomized three-arm study evaluating the combination of bevacizumab with concurrent cFOLFOXIRI and sequential sFOLFOXIRI (c+s FOLFOXIRI; FOLFOX and FOLFIRI alternating every two cycles) compared to FOLFOX. The preliminary results from this trial suggest improved trends for RR and PFS with c+s FOLFOXIRI vs FOLFOX. In the 280 patients in the study, RR was $47 \%$ with FOLFOX/bevacizumab, 60\% with sFOLFOXIRI/bevacizumab (odds ratio [OR] 1.7, 90\% CI 1.05, 2.77; $P=0.075$ ), and $62 \%$ with cFOLFOXIRI/bevacizumab (OR 1.8, 90\% CI 1.12, 2.97; $P=0.040)$. Furthermore, PFS was 11.7 months
(HR 0.672, 90\% CI 0.489, 0.922) with cFOLFOXIRI/bevacizumab, 10.7 months with sFOLFOXIRI/bevacizumab (HR $0.738,90 \%$ CI $0.537,1.012$ ), and 9.3 months with FOLFOX/ bevacizumab. ${ }^{40}$ Additionally, STEAM suggests that the triplet strategy added to bevacizumab may improve the liver resection or $\mathrm{R} 0$ resection rates. The combined results of TRIBE and STEAM suggest that a select group of patients would benefit from triplet chemotherapy plus bevacizumab.

\section{Bevacizumab in patients who are unable to tolerate intensive chemotherapy}

Many patients are not candidates for cytotoxic combination chemotherapy due to comorbidities, poor performance status, age, and/or end organ dysfunction. For this group of patients, treatment guidelines recommend first-line therapy with 5-FU or capecitabine with or without bevacizumab. ${ }^{41}$

Earlier, two Phase II randomized studies evaluating 5-FU in combination with bevacizumab compared to 5-FU alone in patients with untreated mCRC suggested overall improvement in outcome measures with the addition of bevacizumab. ${ }^{29,42}$ Additionally, a recent Phase III study evaluated 407 patients who were randomized to receive capecitabine, capecitabine + bevacizumab, or capecitabine + bevacizumab + mitomycin-c. The findings from this study demonstrated 
an improvement in PFS in all treatment regimens containing bevacizumab vs capecitabine alone. ${ }^{43}$

AVEX is a randomized Phase III study on the combination of bevacizumab and capecitabine vs capecitabine, focusing on an elderly population of patients with mCRC. ${ }^{44}$ In this study, a total of 280 patients were randomized to receive bevacizumab plus capecitabine or capecitabine alone. Patients in the bevacizumab plus capecitabine group had improved PFS (9.1 vs 5.1 months, HR $0.53,95 \%$ CI $0.41-0.69 ; P<0.0001$ ), overall response ( $19 \%$ vs $10 \%, P=0.04)$, and disease control rate $(74 \%$ vs $58 \%, P=0.01) .{ }^{44}$ Additionally, the combination was well tolerated in this patient population.

\section{The role of bevacizumab as maintenance therapy in $\mathrm{mCRC}$}

Given the adverse effect profile of cytotoxic combination chemotherapy, maintenance strategies following induction therapy were designed to maximize benefit while improving the tolerability profile (Table 2 ).

The Phase III MACRO trial is a study where patients were randomized prior to induction therapy to either bevacizumab or XELOX/bevacizumab maintenance after six cycles of XELOX/bevacizumab induction therapy. ${ }^{45}$ In this study, the median OS slightly favored the continuous arm group (23.2 months in the continuous arm vs 20.0 months in the bevacizumab arm, HR 1.05, 95\% CI 0.85-1.30, $P=0.65$ ), although the bevacizumab alone group had less neuropathy ( $8 \%$ vs $26 \%, P<0.0001)$. The SAKK study included patients who did not progress on induction therapy and were randomized to either bevacizumab or drug holiday. ${ }^{46}$ The median time to progression was 4.1 (95\% CI 3.1-5.4) vs 2.9 months (95\% CI 2.8-3.8) and the median OS was 25.4 vs 23.8 months (HR $0.83,95 \%$ CI $0.63-1.1, P=0.2)$ in the bevacizumab and drug holiday groups, respectively.

The STOP and GO trial is another study that evaluated the benefits of maintenance therapy. ${ }^{47}$ The aim of this study was to evaluate maintenance therapy with bevacizumab and capecitabine after induction with XELOX/bevacizumab (Arm B) versus continuing XELOX/bevacizumab (Arm A) until disease progression. PFS was significantly longer in Arm B compared to Arm A (11.0 vs 8.3 months, HR 0.6, $P=0.002$ ), although OS was similar in the two treatment groups $(23.8$ months in Arm B vs 20.2 months in Arm A, $P=0.10$ ).

Another large study, CAIRO-3, evaluated the role of maintenance therapy with bevacizumab plus capecitabine vs drug holiday. ${ }^{48}$ All patients in this study received capectiabine plus oxaliplatin (CapOx)/bevacizumab for induction therapy and then were randomized to receive either bevacizumab/ capecitabine or drug holiday. The median PFS was longer in the maintenance arm compared to the no therapy arm (11.7 vs 8.5 months, HR $0.67,95 \%$ CI $0.56-0.81, P<0.0001)$. Finally, AIO-0207 trial assessed the role of maintenance therapy following induction with 5-FU/oxaliplatin/bevacizumab where patients were randomized to three arms: 1) drug holiday, 2) bevacizumab alone, or 3 ) bevacizumab/capecitabine. ${ }^{49}$ Time to progression was similar in both the maintenance arms and superior to drug holiday.

Collectively, these studies suggest that there is a role of maintenance therapy with a fluoropyrimidine and bevacizumab following induction therapy. Maintenance bevacizumab alone does not seem to be an optimal strategy. Overall, one can conclude that a maintenance strategy is preferable to continuous treatment. In a palliative setting such as this, drug holiday remains an acceptable option to accommodate select patient preferences.

\section{The role of predictive biomarkers}

Several groups have evaluated various angiogenic markers including VEGF-A and other clinical biomarkers to identify predictive and prognostic factors. Recently, a meta-analysis by Zhao et al showed that high VEGF-A levels at baseline were associated with poor $\mathrm{PFS}(\mathrm{HR}=1.26,95 \% \mathrm{CI} 1.12-1.42$, $P=0.0001)$ and $\mathrm{OS}(\mathrm{HR}=1.30,95 \% \mathrm{CI} 1.15,1.46, P<0.0001)$ in comparison to low VEGF-A levels prior to bevacizumab

Table 2 Bevacizumab as maintenance therapy

\begin{tabular}{|c|c|c|c|c|}
\hline Study & Treatment arms & PFS (months) & OS (months) & $P$-value \\
\hline Diaz-Rubio et al ${ }^{45}$ & Bevacizumab vs XELOX + bevacizumab & 10.4 vs 9.7 & 20 vs 23.2 & NS \\
\hline Koeberle et $\mathrm{al}^{46}$ & Bevacizumab vs drug holiday & 4.1 vs 2.9 & 25.4 vs 23.8 & NS \\
\hline Yalcin et $\mathrm{al}^{47}$ & XELOX + bevacizumab vs capecitabine + bevacizumab & 8.3 vs II & 20.2 vs 23.8 & $0.002^{*}$ \\
\hline Simkens et $\mathrm{al}^{48}$ & Bevacizumab + capecitabine vs drug holiday & I I.7 vs 8.5 & 21.6 vs 18.1 & $\begin{array}{l}\text { PFS: }<0.0001 \\
\text { OS: }<0.06\end{array}$ \\
\hline Bennouna et $a^{60}$ & $\begin{array}{l}\text { \#Drug holiday vs } \\
\text { bevacizumab vs bevacizumab + capecitabine }\end{array}$ & 6.9 vs 6.1 & 20.2 vs 21.9 & NS \\
\hline
\end{tabular}

Notes: *Only PFS was significant in this study. \#In this study, there were three groups; however, analysis was done on time to progression for only bevacizumab vs bevacizumab + capecitabine.

Abbreviations: PFS, progression-free survival; OS, overall survival; NS, not significant. 
therapy. ${ }^{50}$ Similarly, another study also showed that patients with lower pretreatment levels of VEGF-A and hepatocyte growth factor experienced an improvement in PFS and OS in comparison to patients with high VEGF-A levels. ${ }^{51}$ This was further validated in prospective trial done by Bruhn et al where the authors demonstrated that high levels of VEGF-A were associated with a poor prognosis due to a shorter PFS (HR $=1.55, P=0.008$ ) compared to low VEGF-A levels. ${ }^{52}$

Furthermore, Uysal et al investigated whether any clinical features in patients who received bevacizumab were associated with patient outcomes. ${ }^{53}$ The authors found that median PFS was 7.2 months in patients with basal proteinuria above $114 \mathrm{mg} /$ day compared to 12 months in those with equal or lower proteinuria $(P=0.01)$. Similarly, PFS was shorter in patients with high lactate dehydrogenase (LDH) or carcinoembryonic antigen (CEA) $(P=0.022$ and $P=0.04$, respectively). However, patients who experienced hypertension were associated with improved clinical outcomes $(P=0.034) .{ }^{53}$ A meta-analysis done by Chen et al reaffirmed that bevacizumab-related hypertension could be potential biomarker for efficacy in mCRC. ${ }^{54}$ Furthermore, Khoja et al also showed that patients who developed proteinuria during therapy have a poorer survival. The median OS was 22.9 months for patients with grade 0 to 1 and 4.2 months for those with grade $\geq 2$ (Table 3 ). ${ }^{55}$

Currently, there are no established predictive molecular or genetic biomarkers to better select patients who may (or may) benefit from anti-angiogenic therapy.

\section{Bevacizumab should not be combined with anti-EGFR therapies in the first-line setting}

Treatment with single targeted agents is often limited secondary to acquired resistance through compensatory activation of alternative signaling pathways. One mechanism to overcome resistance included combining novel agents that target multiple signaling pathways, where preclinical evidence suggests that inhibiting both VEGF and EGFR can

Table 3 Predictive biomarkers for bevacizumab therapy

\begin{tabular}{|c|c|c|c|}
\hline Marker & Study & Outcome & $P$-value \\
\hline \multirow[t]{2}{*}{ Elevated VEGF-A } & Zhao et $\mathrm{al}^{50}$ & Poor PFS and OS & 0.0001 \\
\hline & Bruhn et $\mathrm{al}^{52}$ & Decreased PFS & 0.008 \\
\hline \multirow[t]{2}{*}{ Elevated proteinuria } & Uysal et $\mathrm{al}^{53}$ & Decreased PFS & 0.01 \\
\hline & Khoja et $\mathrm{al}^{55}$ & Decreased OS & 0.028 \\
\hline \multirow[t]{3}{*}{ Hypertension } & Uysal et $\mathrm{al}^{53}$ & Better response & 0.034 \\
\hline & Chen et $\mathrm{al}^{54}$ & Improved PFS and OS & $<0.00001$ \\
\hline & Zhong et al & Improved PFS and OS & $<0.001$ \\
\hline
\end{tabular}

Abbreviations: VEGF-A, vascular endothelial growth factor ligand A; PFS, progression-free survival; OS, overall survival. improve antitumoral activity. ${ }^{56}$ BOND-2, a randomized Phase II trial, investigated the combination of bevacizumab, IFL and cetuximab, or bevacizumab and cetuximab. Patients who received the three-drug combination experienced an absolute 3.1-month survival benefit. ${ }^{57}$

CAIRO-2, which is a randomized Phase III trial, did not confirm the survival benefit of combining bevacizumab with cetuximab. In this study, patients who received dual antibody therapy experienced a worse median PFS in comparison to patients in the bevacizumab arm (9.8 vs 10.7 months; $P=0.019),{ }^{58}$ suggesting that the combination is not more efficacious than with single agents, and may actually lead to worse outcomes. This was consistent with the results of another Phase III study, the PACCE trial, that assessed the role of another EGFR antibody, panitumumab, with bevacizumab and chemotherapy. The panitumumab cohort experienced worse outcomes and a higher level of toxicities. ${ }^{59}$ These two studies suggest that the combination of bevacizumab and an anti-EGFR agent with chemotherapy should not be used in patients treated in first line with mCRC. Ongoing studies continue to evaluate the role of this strategy in more refractory settings.

\section{VEGF vs EGFR inhibitors in the first-line treatment of non-mutated RAS mCRC: which biologic first?}

Numerous studies suggested that agents targeting VEGF or EGFR when added to cytotoxic combination chemotherapy improve outcome in patients with mCRC. The question of which biologic is preferable in the first-line treatment of nonmutated $R A S \mathrm{mCRC}$ has been recently addressed. Heinemann and Stintzing conducted a randomized Phase III trial (FIRE-3) in which patients received FOLFIRI + cetuximab or FOLFIRI + bevacizumab. ${ }^{17}$ There was no significant difference in the primary end point of overall RR (cetuximab: $65.3 \%$ response [95\% CI 58.3-61.1] vs bevacizumab 58\% (95\% CI 52.1-63.7), OR 1.18, 95\% CI 0.85-1.64, $P=0.18)$. The median PFS was similar in both the groups (10 months [95\% CI 8.8-10.8] in the cetuximab group and 10.3 months in the bevacizumab group [HR 1.06, 95\% CI 0.88-1.26; $P=0.55]$ ). Surprisingly, when evaluating $R A S$ WT patients, OS was better in patients who were treated with cetuximab (OS $33.1 \mathrm{vs}$ 25.6 months favoring cetuximab over bevacizumab, $P=0.011$ ).

CALGB 80405 is a large randomized Phase III trial in which patients with KRAS WT tumors received either FOLFIRI or FOLFOX at enrollment and were then randomized to either bevacizumab or cetuximab. ${ }^{60}$ The original trial included unselected patients for $R A S$ status 
who were randomized to either bevacizumab, cetuximab, or both. The findings demonstrated similar results across all the four groups, suggesting that either chemotherapy backbone in combination with either an anti-EGFR or anti-VEGF therapy is an acceptable therapy option in those with $R A S \mathrm{WT}$ tumors (OS: chemotherapy/bevacizumab vs chemotherapy/ cetuximab $=31.2$ vs 32 months [ $P=0.40]$; PFS: chemotherapy $/$ bevacizumab versus chemotherapy/cetuximab $=11.3$ vs 11.4 months $[P=0.31]$ ) (Table 4). In this study, RAS WT patients treated with FOLFIRI with bevacizumab experienced a median OS of 35.2 months in comparison to 32 months in the cetuximab arm $(P=0.7)$.

The collective results from FIRE 3 and CALGB 80405 suggest that addition of bevacizumab or cetuximab confers a similar advantage when combined with chemotherapy in the first-line treatment of patients with mCRC.

\section{The role of bevacizumab beyond progression}

TML 18147 was a multicenter, prospective, randomized, open-label, Phase III trial that evaluated the continuation of bevacizumab following progression on first-line chemotherapy (either oxaliplatin or IFL) with bevacizumab in patients with mCRC. In this study, patients were randomized to receive either bevacizumab with chemotherapy $(\mathrm{N}=409)$ or chemotherapy alone $(\mathrm{N}=411)$. There was an improvement in median OS for patients in the bevacizumab plus chemotherapy group ( 11.1 vs 9.8 months, $P=0.0062) .{ }^{60}$

In addition to bevacizumab, two other anti-angiogenic agents have been approved in mCRC following progression on bevacizumab. The first of these agents is aflibercept (ziv-aflibercept), a fully human recombinant fusion protein that is designed to target VEGF-A, VEGF-B, and placental growth factor. The VELOUR trial is a randomized Phase III study that assessed the role of aflibercept in patients who progressed on prior oxaliplatin therapy and were randomized to receive FOLFIRI with or without aflibercept. Patients who received aflibercept had a significantly longer median OS (13.5 vs 12.06 months, HR 0.817, $P=0.0032) .{ }^{61}$ A subgroup analysis of the $30 \%$ of patients who progressed on bevacizumab confirmed a similar clinical benefit, although the magnitude of survival improvement was less significant. ${ }^{12}$ Unfortunately, the administration of $z i v$-aflibercept to FOLFIRI enhanced cytotoxicrelated toxicities in addition to the expected target specific ones.

The other agent, ramucirumab, is a human monoclonal antibody that targets vascular endothelial growth factor receptor-2. The RAISE trial, a multicenter randomized Phase III study, evaluated the efficacy of ramucirumab in patients with $\mathrm{mCRC}$ as a second-line treatment. Unlike VELOUR, in this study, all patients progressed on bevacizumab in first line. In this trial, a total of 1,072 patients who failed prior treatment with FOLFOX and bevacizumab were randomized to FOLFIRI with or without ramucirumab. There was a significant improvement in both OS and PFS (13.3 vs 11.7 months and 5.7 vs 4.5 months, respectively). ${ }^{62}$

The results of these studies suggest a benefit from continuation of VEGF inhibition following prior exposure to bevacizumab. The effects across all studies are of similar magnitude (Table 5). Given cost and toxicity considerations, bevacizumab beyond progression remains the preferable

Table 4 Anti-VEGF versus anti-EGFR therapy in first-line treatment of non-mutated RAS mCRC

\begin{tabular}{|c|c|c|c|c|c|c|}
\hline & & & PFS (months) & $P$-value & OS (months) & $P$-value \\
\hline \multirow[t]{4}{*}{ KRAS WT } & FIRE-3 ${ }^{17}$ & Cetuximab + FOLFIRI & 10.0 & 0.55 & 28.7 & 0.017 \\
\hline & & Bevacizumab + FOLFIRI & 10.3 & & 25.0 & \\
\hline & CALGB $80405^{63}$ & Cetuximab + FOLFOX/FOLFIRI & 10.4 & 0.55 & 29.9 & 0.34 \\
\hline & & Bevacizumab + FOLFOX/FOLFIRI & 10.8 & & 29.0 & \\
\hline \multirow[t]{4}{*}{ RAS WT } & FIRE-3 $3^{17}$ & Cetuximab + FOLFIRI & 10.4 & 0.54 & 33.1 & 0.0059 \\
\hline & & Bevacizumab + FOLFIRI & 10.2 & & 25.0 & \\
\hline & CALGB $80405^{63}$ & Cetuximab + FOLFOX/FOLFIRI & 11.4 & 0.31 & 32.0 & 0.40 \\
\hline & & Bevacizumab + FOLFOX/FOLFIRI & 11.3 & & 31.2 & \\
\hline
\end{tabular}

Abbreviations: VEGF, vascular endothelial growth factor; EGFR, epidermal growth factor receptor; mCRC, metastatic colorectal cancer; PFS, progression-free survival; OS, overall survival; WT, wild-type.

Table 5 Anti-VEGF after progression with bevacizumab

\begin{tabular}{|c|c|c|c|c|c|}
\hline Study & Treatment arms & Phase & PFS (months) & OS (months) & $\overline{P \text {-value }}$ \\
\hline Bennouna et $a^{60}$ & Second-line chemotherapy (oxaliplatin or irinotecan) + bevacizumab & III & NR & II.I & 0.0062 \\
\hline Van Cutsem et $a^{61 *}$ & FOLFIRI + aflibercept vs FOLFIRI + placebo & III & NR & 13.5 & 0.0032 \\
\hline Tabernero et al $^{62}$ & FOLFIRI + ramucirumab vs FOLFIRI + placebo & III & 5.7 & 13.3 & 0.0219 \\
\hline
\end{tabular}

Note: *Approximately $30 \%$ of patients in this study were exposed to bevacizumab in the first line.

Abbreviations: PFS, progression-free survival; OS, overall survival; NR, not reported. 
anti-angiogenic agent. EGFR inhibitors are acceptable alternatives in the $R A S$ WT group of patients.

\section{Conclusion}

In conclusion, outcome of patients with mCRC has improved significantly over the last two decades and is approaching the 3-year mark. The enhanced outcome has been primarily driven by the expanding armamentarium of agents available to clinics including anti-VEGF therapies such as bevacizumab. Despite improvements in therapy, there remains a noticeable absence of a predictive biomarker to help select patients who are likely to benefit from anti-VEGF therapy.

\section{Disclosure}

The authors report no conflicts of interest in this work.

\section{References}

1. Siegel R, Desantis C, Jemal A. Colorectal cancer statistics, 2014. CA Cancer J Clin. 2014;64(2):104-117.

2. Howlander N, Noone AM, Krapcho M, et al. Previous version: SEER Cancer Statistics Review, 1975-2009 (Vintage 2009 Populations). Updated August 20, 2012. Available from: http://seercancergov/ csr/1975_2009_pops09/.2012. Accessed February 26, 2016.

3. Kuramochi H, Hayashi K, Uchida K, et al. Vascular endothelial growth factor messenger RNA expression level is preserved in liver metastases compared with corresponding primary colorectal cancer. Clin Cancer Res. 2006;12(1):29-33.

4. Tanigawa N, Amaya H, Matsumura M, et al. Tumor angiogenesis and mode of metastasis in patients with colorectal cancer. Cancer Res. 1997;57(6):1043-1046.

5. Kang SM, Maeda K, Onoda N, et al. Combined analysis of p53 and vascular endothelial growth factor expression in colorectal carcinoma for determination of tumor vascularity and liver metastasis. Int J Cancer. 1997;74(5):502-507.

6. Maeda K, Chung YS, Ogawa Y, et al. Prognostic value of vascular endothelial growth factor expression in gastric carcinoma. Cancer. 1996;77(5):858-863.

7. Takahashi Y, Kitadai Y, Bucana CD, Cleary KR, Ellis LM. Expression of vascular endothelial growth factor and its receptor, KDR, correlates with vascularity, metastasis, and proliferation of human colon cancer. Cancer Res. 1995;55(18):3964-3968.

8. Takahashi Y, Tucker SL, Kitadai Y, et al. Vessel counts and expression of vascular endothelial growth factor as prognostic factors in node-negative colon cancer. Arch Surg. 1997;132(5):541-546.

9. Arjaans M, Schroder CP, Oosting SF, Dafni U, Kleibeuker JE, de Vries EG. VEGF pathway targeting agents, vessel normalization and tumor drug uptake: from bench to bedside. Oncotarget. 7(16):2016.

10. Fuchs CS, Tomasek J, Yong CJ, et al. Ramucirumab monotherapy for previously treated advanced gastric or gastro-oesophageal junction adenocarcinoma (REGARD): an international, randomised, multicentre, placebo-controlled, phase 3 trial. Lancet. 2014;383(9911):31-39.

11. Hurwitz H, Fehrenbacher L, Novotny W, et al. Bevacizumab plus irinotecan, fluorouracil, and leucovorin for metastatic colorectal cancer. N Engl J Med. 2004;350(23):2335-2342.

12. Tabernero J, Van Cutsem E, Lakomy R, et al. Aflibercept versus placebo in combination with fluorouracil, leucovorin and irinotecan in the treatment of previously treated metastatic colorectal cancer: prespecified subgroup analyses from the VELOUR trial. Eur J Cancer. 2014;50(2): $320-331$.
13. Ciardiello F, Tortora G. EGFR antagonists in cancer treatment. $N$ Engl J Med. 2008;358(11):1160-1174.

14. Normanno N, Tejpar S, Morgillo F, De Luca A, Van Cutsem E, Ciardiello F. Implications for KRAS status and EGFR-targeted therapies in metastatic CRC. Nat Rev Clin Oncol. 2009;6(9):519-527.

15. Bokemeyer C, Bondarenko I, Hartmann JT, et al. Efficacy according to biomarker status of cetuximab plus FOLFOX-4 as first-line treatment for metastatic colorectal cancer: the OPUS study. Ann Oncol. 2011;22(7): $1535-1546$.

16. Douillard JY, Oliner KS, Siena S, et al. Panitumumab-FOLFOX4 treatment and RAS mutations in colorectal cancer. $N$ Engl J Med. 2013;369(11):1023-1034.

17. Heinemann V, Stintzing S. FOLFIRI with cetuximab or bevacizumab: FIRE-3-authors' reply. Lancet Oncol. 2014;15(13):e583-e584.

18. Lievre A, Bachet JB, Le Corre D, et al. KRAS mutation status is predictive of response to cetuximab therapy in colorectal cancer. Cancer Res. 2006;66(8):3992-3995.

19. Douillard JY, Siena S, Cassidy J, et al. Randomized, phase III trial of panitumumab with infusional fluorouracil, leucovorin, and oxaliplatin (FOLFOX4) versus FOLFOX4 alone as first-line treatment in patients with previously untreated metastatic colorectal cancer: the PRIME study. J Clin Oncol. 2010;28(31):4697-4705.

20. Peeters M, Price TJ, Cervantes A, et al. Randomized phase III study of panitumumab with fluorouracil, leucovorin, and irinotecan (FOLFIRI) compared with FOLFIRI alone as second-line treatment in patients with metastatic colorectal cancer. J Clin Oncol. 2010;28(31):4706-4713.

21. Di Nicolantonio F, Martini M, Molinari F, et al. Wild-type BRAF is required for response to panitumumab or cetuximab in metastatic colorectal cancer. J Clin Oncol. 2008;26(35):5705-5712.

22. De Roock W, Claes B, Bernasconi D, et al. Effects of KRAS, BRAF, NRAS, and PIK3CA mutations on the efficacy of cetuximab plus chemotherapy in chemotherapy-refractory metastatic colorectal cancer: a retrospective consortium analysis. Lancet Oncol. 2010;11(8):753-762.

23. Ciombor $\mathrm{KK}, \mathrm{Wu} \mathrm{C}$, Goldberg RM. Recent therapeutic advances in the treatment of colorectal cancer. Annu Rev Med. 2015;66:83-95.

24. Douillard JY, Cunningham D, Roth AD, et al. Irinotecan combined with fluorouracil compared with fluorouracil alone as first-line treatment for metastatic colorectal cancer: a multicentre randomised trial. Lancet. 2000;355(9209):1041-1047.

25. de Gramont A, Figer A, Seymour M, et al. Leucovorin and fluorouracil with or without oxaliplatin as first-line treatment in advanced colorectal cancer. J Clin Oncol. 2000;18(16):2938-2947.

26. Falcone A, Ricci S, Brunetti I, et al. Phase III trial of infusional fluorouracil, leucovorin, oxaliplatin, and irinotecan (FOLFOXIRI) compared with infusional fluorouracil, leucovorin, and irinotecan (FOLFIRI) as first-line treatment for metastatic colorectal cancer: the Gruppo Oncologico Nord Ovest. J Clin Oncol. 2007;25(13):1670-1676.

27. Gordon MS, Margolin K, Talpaz M, Sledge GW, Jr., Holmgren E, Benjamin R, et al. Phase I safety and pharmacokinetic study of recombinant human anti-vascular endothelial growth factor in patients with advanced cancer. J Clin Oncol. 2001;19(3):843-850.

28. Margolin K, Gordon MS, Holmgren E, et al. Phase Ib trial of intravenous recombinant humanized monoclonal antibody to vascular endothelial growth factor in combination with chemotherapy in patients with advanced cancer: pharmacologic and long-term safety data. $J$ Clin Oncol. 2001;19(3):851-856.

29. Kabbinavar F, Hurwitz HI, Fehrenbacher L, et al. Phase II, randomized trial comparing bevacizumab plus fluorouracil (FU)/leucovorin (LV) with FU/LV alone in patients with metastatic colorectal cancer. J Clin Oncol. 2003;21(1):60-65.

30. Saltz LB, Cox JV, Blanke C, et al. Irinotecan plus fluorouracil and leucovorin for metastatic colorectal cancer. Irinotecan Study Group. $N$ Engl J Med. 2000;343(13):905-914.

31. Hurwitz HI, Fehrenbacher L, Hainsworth JD, et al. Bevacizumab in combination with fluorouracil and leucovorin: an active regimen for firstline metastatic colorectal cancer. J Clin Oncol. 2005;23(15):3502-3508. 
32. Giantonio BJ, Catalano PJ, Meropol NJ, et al. Bevacizumab in combination with oxaliplatin, fluorouracil, and leucovorin (FOLFOX4) for previously treated metastatic colorectal cancer: results from the Eastern Cooperative Oncology Group Study E3200. J Clin Oncol. 2007;25(12):1539-1544.

33. Gustavsson B, Carlsson G, Machover D, et al. A review of the evolution of systemic chemotherapy in the management of colorectal cancer. Clin Colorectal Cancer. 2015;14(1):1-10.

34. Fuchs CS, Marshall J, Mitchell E, et al. Randomized, controlled trial of irinotecan plus infusional, bolus, or oral fluoropyrimidines in first-line treatment of metastatic colorectal cancer: results from the BICC-C Study. J Clin Oncol. 2007;25(30):4779-4786.

35. Hochster HS, Hart LL, Ramanathan RK, et al. Safety and efficacy of oxaliplatin and fluoropyrimidine regimens with or without bevacizumab as first-line treatment of metastatic colorectal cancer: results of the TREE Study. J Clin Oncol. 2008;26(21):3523-3529.

36. Saltz LB, Clarke S, Diaz-Rubio E, et al. Bevacizumab in combination with oxaliplatin-based chemotherapy as first-line therapy in metastatic colorectal cancer: a randomized phase III study. J Clin Oncol. 2008;26(12):2013-2019.

37. Lenz HJ, Lee F, Yau L, et al. MAVERICC, a phase 2 study of mFOLFOX6bevacizumab (BV) vs FOLFIRI-BV with biomarker stratification as firstline (1L) chemotherapy (CT) in patients (pts) with metastatic colorectal cancer (mCRC). J Clin Oncol. 2016;34(suppl 4S; abstr 493).

38. Masi G, Loupakis F, Salvatore L, et al. Bevacizumab with FOLFOXIRI (irinotecan, oxaliplatin, fluorouracil, and folinate) as first-line treatment for metastatic colorectal cancer: a phase 2 trial. Lancet Oncol. 2010;11(9):845-852.

39. Loupakis F, Cremolini C, Masi G, et al. Initial therapy with FOLFOXIRI and bevacizumab for metastatic colorectal cancer. $N$ Engl J Med 2014;371(17):1609-1618.

40. Johanna C, Bendell BRT, James AR, et al; Sarah Cannon Research Institute; Washington University School of Medicine; Florida Cancer Specialists; The Center for Cancer and Blood Disorders; The West Clinic, Memphis; USC Norris Comprehensive Cancer Center; Yale University; Genentech Inc; Duke University Medical Center. Overall response rate (ORR) in STEAM, a randomized, open-label, phase 2 trial of sequential and concurrent FOLFOXIRI-bevacizumab (BEV) vs FOLFOX-BEV for the first-line (1L) treatment (tx) of patients (pts) with metastatic colorectal cancer (mCRC). J Clin Oncol. 2016;34(suppl 4S; abstr 492).

41. Schmoll HJ, Van Cutsem E, Stein A, et al. ESMO Consensus Guidelines for management of patients with colon and rectal cancer. a personalized approach to clinical decision making. Ann Oncol. 2012;23(10):2479-2516.

42. Kabbinavar FF, Schulz J, McCleod M, et al. Addition of bevacizumab to bolus fluorouracil and leucovorin in first-line metastatic colorectal cancer: results of a randomized phase II trial. J Clin Oncol. 2005;23(16):3697-3705.

43. Tebbutt NC, Wilson K, Gebski VJ, et al. Capecitabine, bevacizumab, and mitomycin in first-line treatment of metastatic colorectal cancer: results of the Australasian Gastrointestinal Trials Group Randomized Phase III MAX Study. J Clin Oncol. 2010;28(19):3191-3198.

44. Cunningham D, Lang I, Marcuello E, et al. Bevacizumab plus capecitabine versus capecitabine alone in elderly patients with previously untreated metastatic colorectal cancer (AVEX): an open-label, randomised phase 3 trial. Lancet Oncol. 2013;14(11):1077-1085.

45. Diaz-Rubio E, Gomez-Espana A, Massuti B, et al. First-line XELOX plus bevacizumab followed by XELOX plus bevacizumab or single-agent bevacizumab as maintenance therapy in patients with metastatic colorectal cancer: the phase III MACRO TTD study. Oncologist. 2012;17(1):15-25.

46. Koeberle D, Betticher DC, von Moos R, et al. Bevacizumab continuation versus no continuation after first-line chemotherapy plus bevacizumab in patients with metastatic colorectal cancer: a randomized phase III non-inferiority trial (SAKK 41/06). Ann Oncol. 2015;26(4):709-714.

47. Yalcin S, Uslu R, Dane F, et al. Bevacizumab + capecitabine as maintenance therapy after initial bevacizumab + XELOX treatment in previously untreated patients with metastatic colorectal cancer: phase III 'Stop and Go’ study results--a Turkish Oncology Group Trial. Oncology. 2013;85(6) 328-335.
48. Simkens LH, van Tinteren H, May A, et al. Maintenance treatment with capecitabine and bevacizumab in metastatic colorectal cancer (CAIRO3): a phase 3 randomised controlled trial of the Dutch Colorectal Cancer Group. Lancet. 2015;385(9980): 1843-1852.

49. Hegewisch-Becker S, Graeven U, Lerchenmuller CA, et al. Maintenance strategies after first-line oxaliplatin plus fluoropyrimidine plus bevacizumab for patients with metastatic colorectal cancer (AIO 0207): a randomised, non-inferiority, open-label, phase 3 trial. Lancet Oncol. 2015;16(13): 1355-1369.

50. Zhao L, Zhang D, Ma H, Jin M, Huang F, Zhang T. High VEGF-A level at baseline predicts poor treatment effect of bevacizumab-based chemotherapy in metastatic colorectal cancer: a meta-analysis. Panminerva Med. 2016;58(1):48-58

51. Bai L, Wang F, Zhang DS, et al. A plasma cytokine and angiogenic factor (CAF) analysis for selection of bevacizumab therapy in patients with metastatic colorectal cancer. Sci Rep. 2015;5:17717.

52. Bruhn MA, Townsend AR, Khoon Lee C, et al. Proangiogenic tumor proteins as potential predictive or prognostic biomarkers for bevacizumab therapy in metastatic colorectal cancer. Int J Cancer. 2014;135(3): 731-741.

53. Uysal M, Bozcuk H, Sezgin Goksu S, et al. Basal proteinuria as a prognostic factor in patients with metastatic colorectal cancer treated with bevacizumab. Biomed Pharmacother. 2014;68(4): 409-412.

54. Chen C, Sun P, Ye S, Weng HW, Dai QS. Hypertension as a predictive biomarker for efficacy of bevacizumab treatment in metastatic colorectal cancer: a meta-analysis. J BUON. 2014;19(4):917-924.

55. Khoja L, Kumaran G, Zee YK, et al. Evaluation of hypertension and proteinuria as markers of efficacy in antiangiogenic therapy for metastatic colorectal cancer. J Clin Gastroenterol. 2014;48(5): $430-434$.

56. Shaheen RM, Ahmad SA, Liu W, et al. Inhibited growth of colon cancer carcinomatosis by antibodies to vascular endothelial and epidermal growth factor receptors. Br J Cancer. 2001;85(4):584-589.

57. Saltz LB, Lenz HJ, Kindler HL, et al. Randomized phase II trial of cetuximab, bevacizumab, and irinotecan compared with cetuximab and bevacizumab alone in irinotecan-refractory colorectal cancer: the BOND-2 study. J Clin Oncol. 2007;25(29):4557-4561.

58. Tol J, Koopman M, Cats A, et al. Chemotherapy, bevacizumab, and cetuximab in metastatic colorectal cancer. N Engl J Med. 2009;360(6): 563-572.

59. Hecht JR, Mitchell E, Chidiac T, et al. A randomized phase IIIB trial of chemotherapy, bevacizumab, and panitumumab compared with chemotherapy and bevacizumab alone for metastatic colorectal cancer. J Clin Oncol. 2009;27(5):672-680.

60. Bennouna J, Sastre J, Arnold D, et al. Continuation of bevacizumab after first progression in metastatic colorectal cancer (ML18147): a randomised phase 3 trial. Lancet Oncol. 2013;14(1):29-37.

61. Van Cutsem E, Tabernero J, Lakomy R, et al. Addition of aflibercept to fluorouracil, leucovorin, and irinotecan improves survival in a phase III randomized trial in patients with metastatic colorectal cancer previously treated with an oxaliplatin-based regimen. J Clin Oncol. 2012;30(28): 3499-3506.

62. Tabernero J, Yoshino T, Cohn AL, et al. Ramucirumab versus placebo in combination with second-line FOLFIRI in patients with metastatic colorectal carcinoma that progressed during or after first-line therapy with bevacizumab, oxaliplatin, and a fluoropyrimidine (RAISE): a randomised, double-blind, multicentre, phase 3 study. Lancet Oncol. 2015;16(5):499-508.

63. Venook AP, Niedzwiecki D, Heinz-Josef Lenz, et al. Cancer and Leukemia Group B (Alliance), SWOG, and ECOG. CALGB/SWOG 80405: Phase III trial of irinotecan/5-FU/leucovorin (FOLFIRI) or oxaliplatin/5FU/leucovorin (mFOLFOX6) with bevacizumab (BV) or cetuximab (CET) for patients (pts) with KRAS wild-type (wt) untreated metastatic adenocarcinoma of the colon or rectum (MCRC). J Clin Oncol. 32:5s, 2014 (suppl; abstr LBA3). 


\section{Publish your work in this journal}

Gastrointestinal Cancer: Targets and Therapy is an international, peer-reviewed, open access journal focusing on gastro-intestinal cancer research, identification of therapeutic targets and the optimal use of preventative and integrated treatment interventions to achieve improved outcomes, enhanced survival and quality of life for the cancer patient. The manuscript management system is completely online and includes a very quick and fair peer-review system. Visit http://www.dovepress.com/testimonials.php to read real quotes from published authors.

Submit your manuscript here: https://www.dovepress.com/gastro-intestinal-cancer-targets-and-therapy-journal 